incompletely understood. Making use of an existing dataset, we investigated the CD4+ T-cell transcriptome in early RA patients, seeking biomarkers for drug survival on MTX monotherapy and associated pharmacological insights.

Methods In previous work [1] RNA was rapidly extracted from peripheral blood CD4+ T-cells of 173 early arthritis clinic attendees, at which time patients had been symptomatic for a median of 12 weeks, and were naïve to immunomodulatory treatments. Transcriptional profiling was undertaken using Illumina WG6v3 BeadChip oligonucleotide array technology. For this study a sub-cohort of RA patients was retrospectively identified, whose initial treatment with MTX monotherapy was continued for as long as deemed successful between patient and consulting rheumatologist. Intra-muscular steroid bolus administration (but not oral steroid therapy) was permitted during the study at the discretion of the consulting rheumatologist. MTX monotherapy survival after $\geq 1$ year follow-up (median 28 months) was used as a surrogate outcome for efficacy, and bioinformatics analysis was performed using GeneSpring XI (Agilent).

Results Amongst 31 eligible patients, 19 (61\%) remained on MTX monotherapy at the end of follow-up, but the treatment strategy was unsuccessful (and required modification) for the remaining 12 (39\%). Baseline characteristics and final methotrexate doses were comparable between the two groups. 133 CD4+ T-cell transcripts were identified as being differentially expressed between comparator groups at baseline $(>1.2$ fold-change; $p<0.05)$ and a metric derived from their normalised expression values demonstrated a promising discriminatory utility with respect to MTX monotherapy survival (area under ROC curve 0.91). Functional analysis identified an over-representation of genes involved in apoptosis $(11 / 133$ genes; hypergeometric $p=0.000045)$.

Conclusions Although limited by its reliance on a surrogate efficacy outcome, our pilot study has identified potential transcriptional biomarkers for drug survival on MTX monotherapy amongst early RA patients. Alongside their potential clinical applicability, they suggest that this treatment's efficacy may depend on its ability to regulate CD4+ T-cell survival. Validation amongst a clinically well-characterised, independent early RA cohort is now on-going.

Reference

1. Pratt AG et al, ARD 2012

\section{A3.3 AN IMMUNOLOGICAL WINDOW OF OPPORTUNITY DEFINES THE ABILITY OF EARLY RA PATIENT TO ACHIEVE REMISSION WITH FIRST ANTI-RHEUMATIC TREATMENT}

doi:10.1136/annrheumdis-2013-203216.3

Agata Burska, Rekha Parmar, Yasser El-Sherbiny, Jehan El Jawhari, Paul Emery, Frederique Ponchel. University of Leeds, Leeds, UK

Background and Objectives The therapeutic goal for patients with rheumatoid arthritis (RA) is clinical remission. This is best achieved by early diagnosis followed by appropriate therapeutic intervention. $\mathrm{RA}$ is associated with dys-regulation of T-cell subsets early in the disease with naive cells and regulatory T-cell losses and acquisition of abnormal subset in realtion with inflammation (IRC). Our aim was to test the hypothesis that T-cell subset quantification can predict the achievement of clinical remission in patients with early RA.

Materials and Methods T-cell subsets (naive, Treg and IRC) were quantified using flow cytometry in 108 DMARDs-naïve, early RA patients ( $<24$ months) symptom duration commencing methotrexate MTX or MTX + anti-tumour necrosis factor agents (anti$\mathrm{TNF}$ ) and in 105 healthy controls (HC). The primary outcome assessed was remission (DAS28 $<2.6$ ). The pilot analysis was performed on frozen PBMC obtained from 38 RA patients and $35 \mathrm{HC}$. These results were validated using fresh blood samples on a cohort of 70 RA patients and $70 \mathrm{HC}$.

Results In the pilot study, T-cell subset analysis in early RA confirmed immune dysregulation compared to $\mathrm{HC}$ with reduced frequency of naïve CD4+ T-cells and Treg and increased IRC (all $p<0.001)$ compared to HC. Naive T-cell above median was associated with remission $(p=0.001)$. In the validation study, 50 patients were treated with MTX and showed the same relationship with naïve cell frequency above median being associated with remission $(p=0.011)$. Individual analysis on each patient's naïve cell frequency deviation from expected (using $70 \mathrm{HC}$ ) demonstrated that "normal" naïve cell frequency (observed in 30 patients) was associated with remission whereas reduced naïve cell frequency was more frequently observed in patients with poor response to MTX $(p=0.03)$. Patients with poor immunological status were not prevented to achieve remission when treated with MTX + anti-TNF $(\mathrm{n}=20$ including 10 patients with normal and 10 with reduced naive cells) raising the rate of remission from $20 \%$ in the MTX group ( $\mathrm{n}=4$ of the 20 patients with reduced naïve cells at baseline) to $60 \%$ in the MTX + anti-TNF group ( $n=6$ of the 10 patients).

Conclusions These data show that baseline naïve $\mathrm{T}$-cell subset analysis has a value in predicting early RA MTX treatment outcome. Immunological analysis could be used in conjunction with clinical/serological features to predict response to MTX and select the most appropriate therapy at disease presentation.

\section{A3.4 CD3+CD4-CD8- DOUBLE NEGATIVE TH17 CELLS: NEW INSIGHTS IN THE PATHOGENESIS OF PRIMARY SJÖGREN'S SYNDROME}

doi:10.1136/annrheumdis-2013-203216.4

Alessia Alunno, Sara Caterbi, Onelia Bistoni, Elena Bartoloni, Gianluca Santoboni, Giulia Mirabelli, Francesca Cannarile, Valentina Valentini, Riccardo Terenzi, Roberto Gerli. Rheumatology Unit, Department of Clinical and Experimental Medicine, University of Perugia, Perugia, Italy

Background and Objectives IL-17 axis is widely recognised to be involved in the pathogenesis of autoimmune disorders. Besides conventional $\mathrm{CD}^{+}{ }^{+}$Th17 cells, a small IL-17 producing T-cell population, that lacks of both CD4 and CD8 molecules, defined as double negative (DN) was recently found to be expanded in the peripheral blood (PB) and to accumulate in the kidney in patients with lupus nephritis. Since IL-17 production is enhanced in minor salivary gland (MSG) infiltrates of patients with primary Sjögren's syndrome (pSS), we sought to investigate whether DN T cells may be involved in pSS pathogenesis.

Materials and Methods Thirty patients with pSS and 16 normal controls (NC) were studied. PBMCs were separated by density gradient and phenotypic characterisation was performed by flow cytometry on both freshly isolated cells and after culture (24, 48, 72 and 96 hours). Total PBMCs were cultured in anti-CD3 coated plates in presence or absence of dexamethasone (Dex) at different concentrations. In selected experiments, real time PCR at the same time-points was performed. The study of pSS-MSGs was performed by immunofluorescence.

Results Total circulating DN T cells were increased in pSS compared to NC. NC and pSS freshly isolated DN T cells expressed ROR $\gamma$ t, activation markers (CD25, CD69, HLA-DR) and produced consistent amounts of IL-17. Despite IL-6/TGF $\beta$ ratio became abnormal in pSS patients after 72 hour-culture, Dex was able to down-regulate IL-17 in vitro production in NC and pSS CD4+ Th17 cells and in NC DN T cells from this time-point on. Surprisingly, IL-17 production by pSS-DN T cells was not affected at all by Dex at any time-point. Dex could also reduce the expression of activation markers on $\mathrm{CD}^{+}$cells, but not in pSS and NC-DN T cells. Among DN T cells, those expressing $\alpha \beta T C R$ were expanded in patients with active pSS compared to those with inactive pSS. DN $\mathrm{T}$ cells were present in pSS-MSG infiltrate.

Conclusions To our knowledge, this is the first study identifying and characterising DN T cells in pSS. It shows that DN T cells are 
expanded in the PB of pSS, display an in vivo activated Th17 phenotype, infiltrate MSG and are resistant to corticosteroids. Taken together, these data suggest a key role of this T-cell subset in the perpetuation of chronic sialoadenitis and eventually in SS prognosis and provide the clue to target DN T cells for therapeutic purposes in $\mathrm{pSS}$.

\section{A3.5 COMBINATION BLOCKING OF IL-6 AND IL-21 IN EXPERIMENTAL ARTHRITIS INHIBITS THEIR REDUNDANT ROLE IN TH17-DRIVEN JOINT PATHOLOGY}

doi:10.1136/annrheumdis-2013-203216.5

'Marije I Koenders, 'Debbie M Roeleveld, 1,2Renoud J Marijnissen, ${ }^{3}$ Cheryl NickersonNutter, 'Fons van de Loo, 'Wim B van den Berg. 'Radboud University Nijmegen Medical Centre, Rheumatology Research and Advanced Therapeutics, Nijmegen, the Netherlands; ' University Medical Centre Utrecht, the Netherlands; ${ }^{3}$ Pfizer Inc, Cambridge, Massachusetts, USA

Background and Objectives Both IL- 6 and IL- 21 have been described to drive in vitro Th17 differentiation in the presence of TGFbeta. We explored whether also in vivo IL- 6 and IL-21 play an exchangeable and redundant role in Th17 differentiation during experimental arthritis, and to what extent combined blocking of these cytokines inhibits Th17 differentiation and suppresses arthritis development.

Materials and Methods To investigate the synergistic effects of combined blocking of the IL-6 and IL-21 pathways, arthritis development and Th17 cells were first studied in IL-6 ${ }^{-} \times \mathrm{IL}^{-2} \mathrm{R}^{-1}$ mice and their wild-type (WT) and single-knockout controls during antigen-induced arthritis (AIA). In addition, the kinetic effects of combined IL-6R and IL-21 neutralisation was studied in DBA-1J mice treated at various stages of collagen-induced arthritis (CIA).

Results Mice deficient for either IL-6 or IL-21R showed suppressed antigen-induced arthritis compared to WT controls. This disease reduction was accompanied by a significant reduction in CD4+IL17+ $\mathrm{T}$ cells in the draining lymph nodes as determined by FACS However, mice lacking both the IL- 6 and IL-21 signalling pathways showed an even stronger disease suppression than the IL- $6 \%$ and IL-21 $\mathrm{R}^{-}$mice, and a striking reduction in Th17 levels was observed in these mice.

Based upon our findings in gene-knockout mice, we aimed to confirm the synergistic effects of IL-6/IL-21 with a cytokineneutralisation approach using anti-IL-6R antibodies and sIL-21R-Fc treatment during CIA. Antibodies were given as single treatment or in combination, and was started at immunisation (day 0 ) or around the booster (day 21).

Combined blocking of IL-6R and IL-21 early during arthritis development (day 0) was a very potent approach to prevent arthritis development, reaching a disease incidence of only $40 \%$ at day 35 (isotype control 100\%, sIL-21R-Fc 100\%, anti-IL-6R 60\%). Analyzing the mice that did develop arthritis, we observed that the anti-IL-6R/ sIL-21R-Fc combination was also clearly more potent in suppressing the arthritis severity in comparison to the single treatments. Interestingly, blocking the IL-6/IL-21 pathways at a later stage during arthritis development (day 21) was clearly less effective and did not show any additional effects to anti-IL-6R treatment alone.

Conclusions Combined blocking of the IL- 6 and IL-21 pathways suppresses Th17 differentiation in vivo as demonstrated by our IL-6/IL-21R-deficient mice. However, our neutralisation study during CIA shows that to influence arthritis development this IL-6/ IL-21 blocking approach only has a limited therapeutic window. These findings suggests that to target Th17-driven joint pathology, blocking Th17 effector cytokines like IL-17 and IL-22 might be more effective than attempting to reduce Th17 cell numbers during active disease.

\section{A3.6 COMPARATIVE ANALYSIS OF THE THERAPEUTIC POTENTIAL OF INDUCIBLE TREG CELL POPULATIONS IN EXPERIMENTAL MODEL OF ARTHRITIS}

doi:10.1136/annrheumdis-2013-203216.6

'Julie Quentin, 'Delphine Martire, 'Anne-Laure Mausset-Bonnefont, ${ }^{2}$ Hélène Asnagli, ${ }^{2}$ Nathalie Belmonte, ${ }^{2}$ Arnaud Foussat, 'Christian Jorgensen, 'Rita Vincente, 'Pascale Louis-Plence. 'INSERM U844, Université Montpellier 1, CHU Lapeyronnie, Montpellier, France; ${ }^{2} T x C e l l$, Allée de la Nertière, Les Cardoulines, 06560 Valbonne-Sophia Antipolis, France

Background and Objectives Adoptive cell transfer of Treg cells is a promising approach to restore tolerance in autoimmune disease. However the various type of Tregs, their doses of injection and their in vivo-suppressive mechanism need to be precisely define to clearly establish which Tregs will be able to dampen efficiently the immune response in the various settings.

In our study, we compared the therapeutic potential of IL10secreting Tregs: $\operatorname{Tr} 1$ and CD49b-induced Tregs and $\mathrm{CD}_{2} 5^{+} \mathrm{FoxP} \mathrm{P}^{+}$ Tregs. These two Treg populations share several phenotypic markers as well as immunosuppressive properties. In the present study we perform adoptive cell transfer experiments of the various inducible Treg cells in order to compare their impact on the immune response. Materials and Methods CD49b Treg cells were generated in naive mice following repetitive injections of immature dendritic cells (DC). Cell sorting experiments were realised to obtain $98 \%$ pure CD49b T cells and CD25+ cells. Collagen type II (bCII) specific Tr1 clones were obtained from TCR transgenic mice and expanded in vitro. Selected clones showed in vitro antigen specificity, $\operatorname{Tr} 1$ cytokine profile and IL10- and TGF $\beta$-dependent suppressive activity. Several doses of CD49b or $\operatorname{Tr} 1$ cells were injected i.v. at day 28 in established collagen-induced arthritis. Clinical signs of arthritis were scored, as well as the in vivo impact on the proliferation of Teffector cells and the maturation of DC.

Results We defined for both Treg cell populations the most efficient dose in curative settings experiments. One single dose of $3 \times 10^{6}$ or $1 \times 10^{6}$ of $\operatorname{Tr} 1$ cell administration could reduce the incidence and severity of CIA. Interestingly, higher dose of $10 \mathrm{M}$ of $\operatorname{Tr} 1$ cells did not improve the disease. In the same manner, the dose of $10^{5}$ CD4CD49b ${ }^{+}$cells reverse clinical symptom with a lack of efficacy of higher doses. We demonstrated following injection of Treg cells a clear impact on the proliferation of the effector cells in vivo.

Conclusions Our results suggest that even if the Treg cells present some similarities, we need to precisely define the dose and type of Treg that will be efficient in each experimental setting. We provide also a comparative analysis of the in vivo mechanism responsible of the protection of the various subtypes of Treg cells.

\section{A3.7 COMPARISON OF THE EFFECTS OF TH17 AND TH1 CELLS ON ENDOTHELIAL CELLS AND SYNOVIOCYTES}

doi:10.1136/annrheumdis-2013-203216.7

'Arnaud Hot, 'Laura Maggi, 'Fabien Lavocat, ${ }^{2}$ Francesco Annunziato, 'Pierre Miossec ${ }^{1}$ Department of Clinical Immunology and Rheumatology; Immunogenomic and Inflammation Research Unit EA 4130, Hopital Edouard Herriot, University of Lyon, France; ${ }^{2}$ Department of Internal Medicine and DENOTHE Centre, University of Florence, Firenze, Italy

Background Interaction between different types of T-cells and surrounding non-hematopoietic cells is essential for the proper function of the immune system. Here, we focus on the synoviocytes from RA patients and Endothelial Cells (EC) and compare their interaction with Th1 and Th17 cells.

Methods To assess the interaction of T cells with stromal cells, the effects of Th1 or Th17 cytokines, Th1 or Th17 clone supernatants and coculture of Th clones and stromal cells were analysed. HUVEC (Human Umbilical Vein Endothelial Cells) were used as a model for EC and synoviocytes were isolated from synovium from RA 